\title{
Marco aplicado para la sustentabilidad social y ambiental de fincas productoras de limón (Citrus aurantifolia (Christm) S.) en Portoviejo, Ecuador
}

\section{Applied framework to the social and environmental sustainability of lemon farms (Citrus aurantifolia}

\author{
(christm) S.) in Portoviejo, Ecuador
}

Valarezo-Beltrón Oswaldo $(D)$

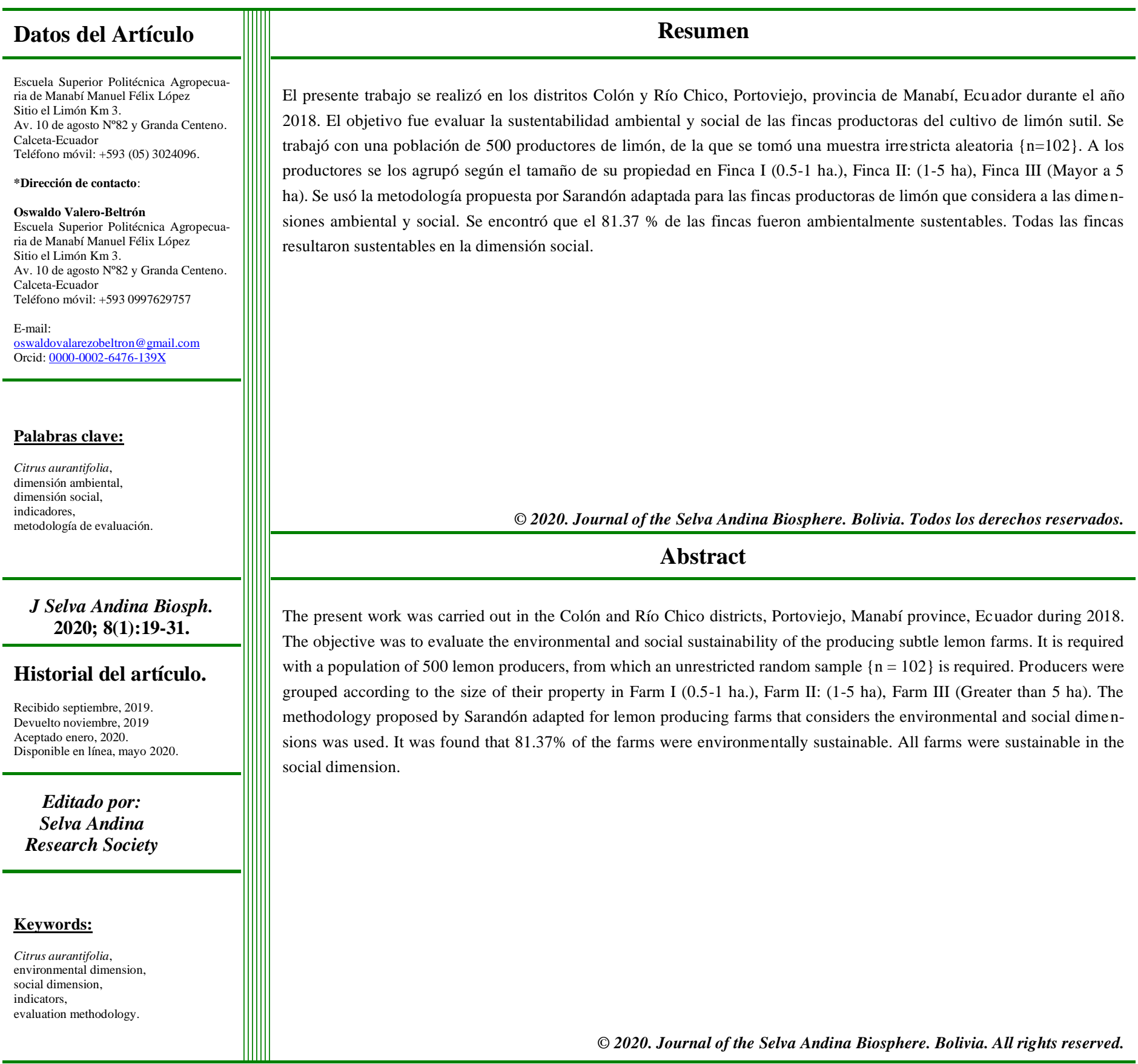




\section{Introducción}

La jurisdicción de Portoviejo es importante por su amplia disponibilidad de recursos productivos, que va desde cultivos de ciclo corto hasta plantaciones perennes, siendo el limón uno de los más relevantes, éste, un producto representativo de la zona, siendo su producción durante años la fuente de ingresos económicos para muchas familias manabitas ${ }^{1}$. Portoviejo, situado en la zona central de la costa de Ecuador, frente al Pacífico Sur, forma parte de la unidad política llamada provincia de Manabí. Los ecosistemas predominantes son, tropical espinoso, situado hacia el borde costero, tropical seco y tropical húmedo, con topografía irregular, desde el nivel del mar hasta los $450 \mathrm{msnm}$, comprende un radio de aproximadamente $100 \mathrm{~km}^{2}$, con gran biodiversidad. La región central costera es la única que no se encuentra articulada al sistema hidrológico de los Andes, dependiendo totalmente del ciclo hidrológico del agua (lluvias regionales) ${ }^{2}$. En su conjunto, las ciudades Manta y Portoviejo actualmente suman un total de 600000 habitantes, aproximadamente 51\% de la población está asentada en pequeñas urbes, el resto en sitios y localidades de carácter rural ${ }^{1}$.

En Ecuador se cultiva limón sutil (Citrus aurantifolia (Christm) Swingle) y el limón Tahití (C. latifolia (Yu. Tanaka) Tan) para el consumo local y exportación, entre ambos existían 4609 ha en 3846 unidades de producción agropecuarias (UPAs) ${ }^{1}$. Según datos más recientes del Ministerio de Agricultura, Ganadería, Acuacultura y Pesca (MAGAP), en el 2008 existían 4377 ha, distribuidas en Manabí (32\%), Pichincha (21\%), Guayas (13\%), Loja (9\%), El Oro (9\%), Imbabura (4\%) y en otras provincias (12\%). El MAGAP calcula que se producen unas 23805 t/año y el rendimiento llega a $4718 \mathrm{~kg} / \mathrm{ha}^{3}$. Desde septiembre hasta finales de noviembre se produce poco, la producción de limón en Manabí se va a mercados de otras provincias, en Mejía y Rio chico se producen cerca de 300 ha de limón y en Santa Ana y Ayacucho 100 ha. Se estima que en la provincia hay unas 600 ha que producen limón ${ }^{4}$. A nivel mundial el precio del limón oscila entre 760 y 860 USD/Tm durante el año $2017^{5}$.

El limón sutil está identificado taxonómicamente con el nombre de $C$. aurantifolia. En nuestro medio se lo conoce como limón criollo o se lo confunde con $C$. limon o limón verdadero, tal vez por el alto contenido de ácido y su nombre común, especialmente en países de habla hispana ${ }^{6}$. Pertenece a la familia de las rutáceas, tiene nombres vulgares como lima, lima ácida, criollo, sutil o limón agrio Esta especie es nativa del Asia, pero crece muy bien en las tierras bajas del trópico pues necesita altas temperaturas para un buen desarrollo ${ }^{8}$.

Asegurar la alimentación de una población humana creciente, con criterios de sostenibilidad y ante la amenaza del cambio climático son los retos principales de la agricultura del siglo $\mathrm{XXI}^{9}$. El ingeniero Agrónomo debe transformarse en un gestor cultural, en un agente de cambio, la sostenibilidad ambiental debe encontrar en la agricultura un apoyo que vaya más allá de su compromiso, con el futuro alimentario de la humanidad ${ }^{10}$. La ciencia de la sostenibilidad surge para comprender el sistema, cada vez más complejo constituido por las sociedades humanas y los sistemas naturales con los que interactúan ${ }^{11}$. La sustentabilidad es la habilidad de lograr una prosperidad económica sostenida en el tiempo protegiendo al mismo tiempo los sistemas naturales del planeta y proveyendo una alta calidad de vida para las perso$\operatorname{nas}^{12}$.

Existen diferentes metodologías para evaluar la sustentabilidad en la agricultura entre ellas se encuentran modelos en el ámbito de unidades de producción, modelos integrales y modelos de presiónestado-respuesta. A nivel mundial se ha trabajado 
bastante en los modelos en el ámbito de unidades de producción, las que sobresalen el marco de Sarandón que comprende la dimensión social, ambiental y económica ${ }^{13}$. También se señala al Marco de Evaluación de Sistemas de Manejo incorporando Indicadores de Sustentabilidad (MESMIS) que se dirigen a proyectos agrícolas, forestales y pecuarios llevados a cabo colectiva o individualmente y que se orientan al desarrollo y/o la investigación ${ }^{14}$.

Para que la agricultura sea sostenible es muy importante, para que mantenga las necesidades actuales y futuras, debe proteger y mejorar la calidad del aire, suelo y agua, es decir debe ser amigable con el medio ambiente, pero para ello es indispensable partir de una línea base de los diferentes sistemas agrícolas que se logra con su diagnóstico. Los marcos de evaluación de la sustentabilidad equivalen a una foto de la realidad en determinado momento, constituyen el punto de partida para determinar los puntos débiles. Además, cuando se cuenta con un diagnóstico se puede recurrir a entidades públicas y privadas para financiar actividades a favor de la sustentabilidad de un agroecosistema, como es el caso del limón.
Por lo expuesto se planteó como objetivo evaluar la sustentabilidad ambiental y social de las fincas productoras del cultivo de limón Sutil (C. aurantifolia (Christm) Swingle) en Portoviejo, Ecuador.

\section{Materiales y métodos}

El presente trabajo se realizó en los distritos Colón y Río Chico, cantón Portoviejo, provincia de Manabí, Litoral de Ecuador. Se trabajó con una población de 500 productores de limón, de la que se tomó una muestra irrestricta aleatoria $\{\mathrm{n}=102\}^{15}$. A los productores se los agrupó según el tamaño de su propiedad en Finca I (0.5-1 ha.), Finca II: (1-5 ha), Finca III (Mayor a 5 ha). Cada grupo de Finca abarcó 34 unidades productivas (UP).

Para evaluar la sustentabilidad se usó la metodología propuesta por Sarandón et $\mathrm{al}^{16}$, pero adaptada para las fincas productoras de limón (FPL) que considera las dos dimensiones de la sustentabilidad (ambiental y social), el procedimiento fue el siguiente:

Tabla 1 Selección y construcción de sub-indicadores y variables usadas para evaluar la sustentabilidad de fincas productoras de limón. (Adaptado de Sarandón et al. ${ }^{16}$ )

\begin{tabular}{|c|c|c|}
\hline Dimensión & Sub-indicadores & Variables \\
\hline \multirow{3}{*}{$\begin{array}{l}\text { Dimensión ambiental. (IA) } \\
\text { un sistema será ecológicamente } \\
\text { sustentable si conserva la base de los } \\
\text { recursos productivos y disminuye el } \\
\text { impacto sobre los recursos extra } \\
\text { prediales }\end{array}$} & A. Conservación de la vida del suelo. & $\begin{array}{l}\mathrm{A}_{1}-\text { Manejo de la cobertura vegetal. } \\
\mathrm{A}_{2} \text { - Diversificación de Cultivos }\end{array}$ \\
\hline & B. Riesgo de erosión. & $\begin{array}{l}\mathrm{B}_{1} \text { - Pendiente predominante. } \\
\mathrm{B}_{2} \text { - Conservación de suelos. }\end{array}$ \\
\hline & C. Manejo de la Biodiversidad & $\mathrm{C}_{1}$-Área de zonas de Conservación \\
\hline \multirow{3}{*}{$\begin{array}{l}\text { Dimensión Sociocultural (ISC) } \\
\text { para conocer grado de satisfacción } \\
\text { de los aspectos socioculturales. }\end{array}$} & $\begin{array}{l}\text { A. Satisfacción de las necesidades } \\
\text { básicas. }\end{array}$ & $\begin{array}{l}\mathrm{A}_{1}-\text { Vivienda. } \\
\mathrm{A}_{2} \text { - Acceso a la educación. } \\
\mathrm{A}_{3} \text { - Acceso a salud y cobertura sanitaria. } \\
\mathrm{A}_{4}-\text { Servicios Básicos }\end{array}$ \\
\hline & \multicolumn{2}{|l|}{ B. Integración social. } \\
\hline & \multicolumn{2}{|l|}{$\begin{array}{l}\text { C. Conocimiento Tecnológico y } \\
\text { Conciencia Ecológica. }\end{array}$} \\
\hline
\end{tabular}

a.-Selección y construcción de sub-indicadores: Se seleccionaron y construyeron de acuerdo a la meto- dología y el marco conceptual propuesto por Sarandón et $\mathrm{al}^{17}$, pero adaptada para el cultivo de limón, 
ya que se considera que la propuesta original está diseñada para fincas que trabajan con cultivos anuales y no para aquellas que tienen cultivos permanentes (tabla 1$)^{18}$. Para esta actividad se planteó preliminarmente los indicadores y variables en la Escuela Superior Politécnica Agropecuaria de Manabí (ESPAM), luego se los mejoró en conjunto con los propietarios y profesionales relacionados a la producción del cultivo de limón (CL) y funcionarios de organismo públicos Instituto Nacional de Investigación Agropecuarias (INIAP, Municipalidad de Portoviejo), representantes de organizaciones de productores como la Junta de Riego de Riochico y Asociación de Productores de Coco, Limón y Cacao (APROLIC).

b. Estandarización y ponderación de los indicadores: Para realizar las comparaciones entre fincas, los datos obtenidos para cada variable fueron estandarizados a una escala sencilla de 0 a 4 . El valor 4, representa la mayor sustentabilidad y 0 la más baja sustentabilidad. Posteriormente, los valores obtenidos para cada variable o sub-indicador, se ponderaron multiplicándolos por un coeficiente de acuerdo a la importancia relativa de cada variable respecto a la sustentabilidad. Si el valor es dos o mayor a dos se considera como sustentable. Una vez finalizada la ponderación se procedió a aplicar a los productores los indicadores y variables relacionados con la sustentabilidad social y ambiental ${ }^{16}$.

\section{Dimensión ambiental.}

A.-Conservación de la vida de suelo. Un sistema es sustentable si las prácticas mantienen o mejoran la vida en el suelo. Para construir este indicador se consideró lo siguiente:

$A_{l .}$-Manejo de la cobertura vegetal (MCV). La misma provee al suelo de una protección contra los agentes climáticos y disminuye el riesgo de erosión. Los valores son (4) $100 \%$ de cobertura (3) 99 a 75 $\%$ (2): 74 a $50 \%$ (1) 50 a $25 \%$; (0) menos del 25 $\%^{17}$.
$A_{2 .}$-Diversificación de cultivos (DC). Se valoró de la siguiente manera (4) totalmente diversificado, con asociaciones de cultivos y con vegetación natural (3) alta diversificación de cultivos, con asociación media entre ellos (2) diversificación media, con muy bajo nivel de asociación entre ellos (1) poca diversificación de cultivos, sin asociaciones (0) monocultivo ${ }^{17}$.

B.-Riesgo de erosión (RE). Un sistema es sustentable si logra minimizar o evitar la pérdida de suelo debido a la erosión hídrica. Se consideró las siguientes variables:

$B_{1}$-Pendiente predominante $(P P)$. Se valoró de la siguiente manera (4) 0 al $5 \%$ (3) 6 al $10 \%$ (2) 11 al $15 \%$ (1) 16 al $20 \%$ (0) más de $20 \%$. Los suelos tienen una ligera pendiente como generalidad ${ }^{17}$.

$B_{2}$-Conservación del suelo $(C S)$. El sistema sustentable es aquel que conserva la cantidad y calidad de sus suelos. Se considera las prácticas usadas para la conservación de suelos en la finca y su valoración fue la siguiente (4) Plantación en curvas de nivel o terrazas (3) Barreras vivas (2) Barreras muertas (1) Plantación en tresbolillo (0) Plantación en sentido de la pendiente ${ }^{17}$.

C.-Manejo de la Biodiversidad (MD). La biodiversidad es importante para la regulación del sistema ya que, entre otras funciones, proporciona hábitat y nichos ecológicos para los enemigos naturales. El efecto del sistema de manejo de la finca sobre la biodiversidad, se evaluó a través del siguiente componente:

$C_{1}$-Área de zonas de conservación (AZC). Las zonas de conservación incluyen bosques, pastizales, pantanos, orillas de ríos y riachuelos, zonas de amortiguamiento, donde no se realice labores agrícolas y por el contrario estén adecuadamente delimitadas y conservadas. Se empleó la siguiente escala (4) mayor de 10\%; (3) 10 a 7\%; (2) 6 a 3\%; (1) 3-1\%; (0) menor a $1 \%$ en área de conservación del total de superficie de la finca ${ }^{17}$. 
El indicador que mide el grado de cumplimiento de la dimensión ambiental (IA), se calculó de la siguiente manera ${ }^{17}$ :

Indicador ambiental $(\mathrm{IA})=\underline{(\mathrm{A} 1+\mathrm{A} 2) / 2+(\mathrm{B} 1+\mathrm{B} 2) / 2+(\mathrm{C} 1) / 1}$ 3

\section{Dimensión Social.}

A.-Satisfacción de las necesidades básicas (SNB). Un sistema sustentable si los agricultores tienen aseguradas sus necesidades básicas que comprenden educación, salud, vivienda y servicios básicos. Está compuesto por los siguientes sub-indicadores:

$A_{1}$-Vivienda, la valoración fue de la siguiente manera (4) casa de hormigón (3) casa mixta (2) casa de madera (1) casa de caña (0) no tiene casa propia ${ }^{17}$.

$A_{2}$-Acceso a la educación (AE), la valoración se realizó de la siguiente manera (4) Acceso a educación superior (3) Acceso a educación secundaria (2) Acceso a educación primaria y secundaria con restricciones (1) Acceso a educación primaria (0) Sin acceso a la educación ${ }^{17}$.

$A_{3}$-Acceso a salud y cobertura sanitaria (ASCS). Se refiere a la distancia en $\mathrm{km}$ desde la finca al centro médico más cercano donde se pueda atender emergencias médicas y se gestione el traslado a centros más complejos. La valoración se cumplió de la siguiente manera (4) menos de $1 \mathrm{~km} \mathrm{(3)} 1.1 \mathrm{a} 3 \mathrm{~km}$ (2) 3.1 a $5 \mathrm{~km}$ (1) 5.1 a $10 \mathrm{~km}$ (0) más de $10 \mathrm{Km}^{17}$. $A_{4}$-Servicios. La valoración de los servicios en casa, fue de la siguiente manera (4) Instalación completa de agua potable, desagüe y electricidad (3) Instalación de agua potable y desagüe (2) Instalación de electricidad y agua entubada (1) Sin instalación de luz y agua entubada (0) Sin luz y sin fuente de agua cercana $^{17}$.

B.-Integración social (IS). Se evaluó la relación con otros miembros de la comunidad, nivel de participación en las organizaciones de su ámbito de acción, considerando la actitud de liderazgo. Se valoró (4) como Muy alta (3) Alta (2) Media (1) Baja (0) Nu$1 \mathrm{a}^{17}$.

C.-Conocimiento tecnológico y conciencia ecológica (CTCE). El conocimiento tecnológico y la agricultura sustentable son fundamentales para tomar decisiones adecuadas respecto a la conservación de los recursos y mantener o mejorar los sistemas productivos. La valoración fue de la siguiente manera: (4) Aplica totalmente en su plantación un manejo integrado del cultivo (3) Aplica mayoritariamente en su plantación un manejo integrado del cultivo (2) Aplica medianamente en su plantación un manejo integrado del cultivo (1) Aplica minoritariamente en su plantación un manejo integrado del cultivo (0) No aplica en su plantación un manejo integrado del cultivo ${ }^{17}$.

Para la dimensión social, se considerará de mayor peso a los indicadores de satisfacción de necesidades básicas y la fórmula se la calculó de la siguiente forma ${ }^{17}$ :

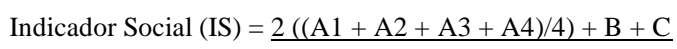

\section{Resultados}

Cuando se evaluó la sustentabilidad ambiental (SA), $81.37 \%$ de las fincas evaluadas tuvieron un IA $<2$, más de $75 \%$ del total de FPL son ecológicamente insostenibles. Las variables relacionadas con la conservación de la vida del suelo (A), como MCV $\left(A_{1}\right)$ y DC de las fincas $\left(A_{2}\right)$ y en $C_{1}$ que hace referencia a las áreas dedicadas a la conservación alcanzaron los valores más bajos tabla 2 .

Para la Finca I, tres de los cinco rubros en los fundos poseen valores menores que 2 , obteniendo un grado de sustentabilidad bajo (1.49), con los subindicadores MCV (0.96), DC (1.04), PP (0.81) tabla 3, figura 1 . 
Tabla 2 Resumen de la evaluación de la sustentabilidad ambiental en fincas productoras de limón

\begin{tabular}{|c|c|c|c|c|c|c|c|}
\hline \multirow[b]{3}{*}{ Variables } & \multicolumn{5}{|c|}{ Subindicadores } & \multirow{2}{*}{\multicolumn{2}{|c|}{ IA }} \\
\hline & & 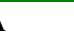 & & & $\mathrm{C}$ & & \\
\hline & $\mathrm{A}_{1}$ & $\mathrm{~A}_{2}$ & $\mathrm{~B}_{1}$ & $\mathrm{~B}_{2}$ & $\mathrm{C}_{1}$ & Mayor a 2 & Menor a 2 \\
\hline Promedio & 0.94 & 0.98 & 3.11 & 2.33 & 0.84 & $18.63 \%$ & $81.37 \%$ \\
\hline
\end{tabular}

La Finca II también no superó el grado objetivo de sustentabilidad, alcanzó un promedio global de 1.62, cabe recalcar que en los subindicadores MCV (A1), DC $\left(\mathrm{A}_{2}\right)$ y $\mathrm{AZC}\left(\mathrm{C}_{1}\right)$ los valores fueron los más bajos, tabla 3 , figura 1 .

Figura 1 Resumen de la Dimensión ambiental de las tres fincas tipo

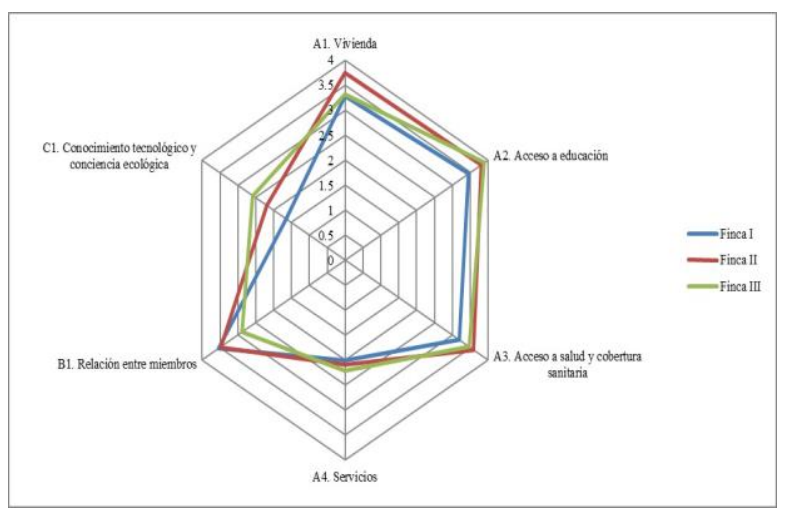

En la Finca III, sólo dos de los cinco subindicadores resultaron tener un valor mayor a dos, exceptuando los subindicadores $\mathrm{MCV}\left(\mathrm{A}_{1}\right), \mathrm{DC}\left(\mathrm{A}_{2}\right)$ y $\mathrm{AZC}\left(\mathrm{C}_{1}\right)$, lo que significó que no sea sustentable con un índice de 1.92. Cabe señalar que la Finca III alcanzó el valor más alto en la escala entre las fincas tipo. tabla 3 , figura 1 .

En la tabla 4, $100 \%$ de las fincas evaluadas tuvieron un ISC mayor a 2, lo que significa que el sistema de producción es totalmente sustentable. Las variables asociadas a la SNB (A) como tipo de vivienda $\left(\mathrm{A}_{1}\right), \operatorname{AE}\left(\mathrm{A}_{2}\right)$, a $\operatorname{ASCS}\left(\mathrm{A}_{3}\right)$, tienen valores mayores a 3 , sucediendo lo mismo con la integración social $\left(\mathrm{B}_{1}\right)$.
La tabla 5 y figura 2 muestran los valores para la dimensión social, todos los indicadores tienen un valor mayor a 2 , pues se observan valores altos en todos los subindicadores, excepto en la Finca I para C1 que sólo llega a 1.65. Las tres Fincas tipo son sustentable en el aspecto sociocultural, y la Finca II, tiene los valores más altos comparado con las otras fincas, alcanzado un grado de sustentabilidad de 3.16 .

\section{Discusión}

La sustentabilidad de las actividades en el mundo es un tema que está en boga en la actualidad porque los recursos cada día son más escasos. La agricultura no es ajena a esta situación, de hecho muchas áreas de cultivo en el mundo han desaparecido debido a un manejo erróneo impulsado por la revolución verde. La agricultura sustentable es una actividad agropecuaria que se apoya en un sistema de producción que tenga la aptitud de mantener su productividad y ser útil a la sociedad a largo plazo, cumpliendo los requisitos de abastecer adecuadamente de alimentos a precios razonables y ser suficientemente rentable como para competir con la agricultura convencional, y además, el ecológico de preservar el potencial de los recursos naturales productivos. Se caracteriza por el mejoramiento y la conservación de la fertilidad, la productividad del suelo con estrategias de manejo (insumos de bajo costo) ${ }^{19-22}$. Busca la minimización de los impactos, protección y mejoramiento del ambiente ${ }^{23}$. Las prácticas que se promueven son los cultivos tradicionales, abonos verdes, rota- 
ción de cultivos, integración de sistemas agrícolas-

pecuarios, sistemas agropforestales ${ }^{24}$.

Tabla 3 Resumen de la evaluación de la sustentabilidad ambiental en fincas tipos productoras de limón

\begin{tabular}{llll}
\hline Indicador & Finca I & Finca II & Finca III \\
\hline $\mathrm{A}_{1}$. Manejo cobertura vegetal & 0.96 & 0.89 & 1.05 \\
$\mathrm{~A}_{2}$. Diversificación cultivos & 1.04 & 0.89 & 1.16 \\
$\mathrm{~B}_{1}$. Pendiente predominante & 0.81 & 3.88 & 3.95 \\
$\mathrm{~B}_{2}$. Conservación de suelo & 2.31 & 2.33 & 2.37 \\
$\mathrm{C}_{1}$. Área de zonas de conservación & 2.31 & 0.11 & 1.05 \\
\hline Total & 7.43 & 8.10 & 9.58 \\
Promedio & 1.49 & 1.62 & 1.92 \\
Cumplimiento & No & No & No \\
\hline Conservación de la vida de suelo (A) Riesgo de erosión (B) Manejo de la Biodiversidad (C)
\end{tabular}

Tabla 4 Resumen de la evaluación de la sustentabilidad social en fincas productoras de limón

\begin{tabular}{|c|c|c|c|c|c|c|c|c|}
\hline \multirow[b]{3}{*}{ Variables } & \multicolumn{6}{|c|}{ Subindicadores } & \multirow{2}{*}{\multicolumn{2}{|c|}{ ISC }} \\
\hline & & & & & B & $\mathbf{C}$ & & \\
\hline & $\mathrm{A}_{1}$ & $\mathrm{~A}_{2}$ & $\mathrm{~A}_{3}$ & $\mathrm{~A}_{4}$ & $\mathrm{~B}_{1}$ & $\mathrm{C}_{1}$ & Mayor a 2 & Menor a 2 \\
\hline Promedio & 3.54 & 3.75 & 3.47 & 2.09 & 3.39 & 2.13 & $100 \%$ & $0 \%$ \\
\hline
\end{tabular}

(A) Satisfacción de las necesidades básicas, (B) Integración Social, (C) Conocimiento y conciencia ecológica.

Tabla 5 Resumen de la evaluación de la sustentabilidad social en fincas tipos productoras de limón

\begin{tabular}{llll}
\hline Indicador & Finca I & Finca II & Finca III \\
\hline A $_{1}$ Vivienda & 3.27 & 3.74 & 3.32 \\
A2. Acceso a educación & 3.46 & 3.82 & 3.89 \\
A3. Acceso a salud y cobertura sanitaria & 3.19 & 3.6 & 3.47 \\
A4. Servicios & 2 & 2.09 & 2.21 \\
B1. Relación entre miembros & 3.54 & 3.49 & 2.89 \\
C1. Conocimiento tecnológico y conciencia ecológica & 1.65 & 2.19 & 2.58 \\
Total & 17.11 & 18.93 & 18.36 \\
Promedio & 2.85 & 3.16 & 3.06 \\
Cumplimiento & Sí & Sí & Sí \\
\hline
\end{tabular}

Satisfacción de las necesidades básicas (A) Integración Social (B) Conocimiento Tecnológico y Conciencia Ecológica (C).

Figura 2 Resumen de la Dimensión Social de las tres fincas tipo

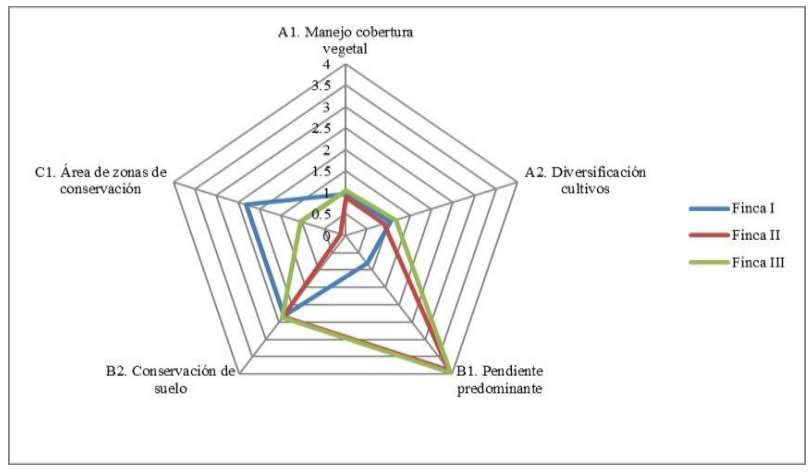

La agricultura interactúa en forma permanente con el medio ambiente y, por definición, lo artificializa para dirigirlo hacia la producción de bienes para el ser humano ${ }^{25}$. La producción agropecuaria puede generar transformaciones importantes en el medio ambiente: en algunos casos son fuente de contaminación del agua por nitratos, fosfatos y plaguicidas $^{26}$. La actividad es una fuente emisora de gases responsables del efecto invernadero, metano y óxido nitroso, contribuyendo en gran medida a otros tipos de contaminación del aire y del agua ${ }^{27}$. Determina- 
dos métodos agrícolas y forestales son la principal causa de la pérdida de biodiversidad del mundo ${ }^{28}$. El objetivo de la presente investigación se cumplió según lo planificado al determinar la sustentabilidad ambiental y social. No obstante, los principales hallazgos se recalca que la SA del proyecto en el cantón Portoviejo no es favorable, ya que la mayoría de las fincas son insostenibles, $81.37 \%$ tuvieron un IA menor a 2. También un hallazgo alentador fue que la sostenibilidad social del cultivo en la zona fue óptima, lo que significa que el sistema de producción es socialmente sustentable.

El sistema de producción agroecológico es ambientalmente más sustentable que los sistemas de producción convencional y en transición a agroecológi$\mathrm{co}^{29}$. Un sistema de producción amigable con el ambiente enfatiza en la conservación de los recursos naturales, en la preservación de la biodiversidad, en el uso de insumos de origen biológico para el control de plagas y enfermedades y hay una interacción hombre-naturaleza ${ }^{30-32}$. En investigaciones previas, el sistema de producción orgánica alcanzó un mayor Indicador General Ambiental ( $\mathrm{IA}=2.71$ ), más alto que el que alcanzó el sistema de producción convencional $(\mathrm{IA}=2.08)^{33}$. En FPL la dimensión ambiental alcanzó un valor promedio de $2.09^{34}$. En Santa Elena, Ecuador el $57.8 \%$ de las fincas evaluadas tuvieron un IA mayor a 2 , es decir poco más de la mitad de FPL son ambientalmente sustentables ${ }^{35}$.

Al analizar el diagnóstico de la sostenibilidad ambiental de las FPL se establece que es preocupante, lo que representa a mediano y largo plazo problemas en lo referente a la naturaleza del agroecosistema. Por lo general para el MCV muchos productores emplean herbicidas que eliminan plantas pequeñas que albergan diversidad de interacciones entre seres vivos. Los agricultores tienen una disyuntiva al elegir los cultivos que quieren establecer en sus predios, casi siempre terminal eligiendo con base en el aspecto económico, quedando la parte ambiental de lado, por lo que se tiende hacia el monocultivo ya que pequeñas extensiones de una plantación generan dificultades en la comercialización.

Cuando se habla de sostenibilidad social se está poniendo el enfoque sostenible en la forma de vida de un grupo social en concreto $^{36-39}$. Este tipo de sostenibilidad es especialmente importante en el caso de pueblos y sectores sociales que cuentan con una situación de desprotección o de desventaja con respecto a los demás ${ }^{40-43}$. Su principal objetivo es garantizar que las actividades humanas se puedan desarrollar de tal modo que no destruyan las comunidades humanas a las que afecta, lo que garantiza la perdurabilidad de las mismas y de sus estilos de vida a largo plazo $^{44,45}$. El practicar una agricultura sustentable garantiza la conservación y bienestar de nuestras comunidades rurales ${ }^{46,47}$. Cuando se tiene una agricultura tradicionalista que no respete el entorno y destruya los recursos será el ente que siga expulsando a los trabajadores del sector primario a las zonas urbanas ${ }^{48,49}$.

Diversas investigaciones establecen que agroecosistemas de Perú fueron socialmente sustentables, ya que en todas las fincas analizadas el ISC fue superior a dos 50. En fincas de la costa sur de Ecuador la dimensión social, fue la que presentó la valoración más alta, con 2.66 ${ }^{51}$. En fincas Argentina la mayoría superó el valor de dos. Casi siempre cuando existe un manejo adecuado de los recursos naturales en una plantación la parte social es positiva también ${ }^{52}$. La sostenibilidad social de este del proyecto se muestra alentadora para las futuras generaciones al presentar todos los subindicadores mayores a 2, dejando en claro que en la jurisdicción analizada las relaciones entre los miembros se encuentran muy cohesionadas. Dentro del medio existen algunas asociaciones productivas que refuerzan las interacciones humanas entre los agricultores. La situación de la zona está en concordancia con otros lugares 
del mundo ya que presentan valores favorables hacia los actores sociales de las comunidades agropecuarias.

Como aspectos principales del trabajo de investigación se concluye lo siguiente:

i).-Mediante la evaluación de la sustentabilidad ambiental aplicada a las tres FPL de Portoviejo, $81.37 \%$ de las fincas tuvieron un IA menor a 2 , es decir más de $75 \%$ del total de fincas productoras de limón son ecológicamente insostenibles. Para lo cual es fundamental buscar alternativas de producción que disminuya estos impactos negativos a los que se expone el ecosistema y lograr un sistema eficiente y estable en el tiempo.

ii).-En cuanto a la evaluación de la sustentabilidad social en las tres fincas se detectó que el $100 \%$ de ellas tienen un indicador sociocultural mayor a 2, dando como resultado que cuentan con un sistema de producción sustentable, dado que las variables asociadas a la SNB como vivienda, educación, salud, cobertura sanitaria e integración social tienen valores mayores a tres.

iii).-Los valores obtenidos muestran que se debe encaminar esfuerzo hacia lograr un mejor desarrollo de las plantaciones y del entorno agrícola de las FPL, a través de procesos sistemáticos de manejo para lograr la sostenibilidad de los recursos, para ello es necesario que se efectúen investigaciones y se diseñen acciones productivas y de cambio social que mejoren el nivel de vida de los productores y contribuyan al logro de la sustentabilidad en las dimensiones económicas, sociales y ambientales.

\section{Fuente de financiamiento}

El trabajo fue financiado en parte por la Escuela Superior Politécnica Agropecuaria de Manabí a través de fondos del Ministerio de Finanzas del Ecuador, los cuales son destinados a investigación 27 por el Gobierno Nacional de la República del Ecuador. Algunas actividades se cubrieron con financiamiento del autor

\section{Conflictos de intereses}

El autor declara que no tienen conflictos de interés con la presente investigación.

\section{Agradecimientos}

Se agradece a Dirección de carrera de Administración de Empresas con mención en agropecuaria y agroindustria y a la Coordinación General de Investigación pertenecientes a la Escuela Superior Politécnica Agropecuaria de Manabí; y, al Programa de Agricultura Sustentable de la Universidad Nacional Agraria La Molina. También va mi profundo agradecimiento a las autoridades originarias distritos Colón y Río Chico, cantón Portoviejo.

\section{Aspectos Éticos}

Todos los aspectos procedimentales experimentales fueron enmarcados y aprobados según el Código de Ética perteneciente a la Escuela Superior Politécnica Agropecuaria de Manabí.

\section{Literatura Citada}

1. Instituto Nacional de Estadísticas y Censos. Resultados Nacionales y Provinciales. Quito: Editorial INEC; 2016. 264 p.

2. Briones G. La cuenca del Río Portoviejo. Portoviejo: Editorial Utm; 2002. 59 p.

3. Agronegocios [Internet]. El limón se adapta incluso a la altitud. 2010 [citado el 14 de mayo de 2015]. Recuperado a partir de: http://agronegociosecuador.ning.com/notes/El_li m\%C3\%B3n_se_adapta_incluso_a_la_altitud 
4. Diario Manabita. El limón cuesta el triple en los mercados por falta de producto. Diario Manabita (Portoviejo). 29 de Septiembre de 2014;12.

5. Indexmundi [Internet]. Índice de precios agrícolas en el mundo. 2017 [citado 9 de Julio de 2019]. Recuperado a partir de: http://www.indexmundi.com/es/precios-demercado/?mercancia=limones

6. Whiteside JP. Compedium of citrus diseases. Aps Press. New York-EEUU; 2009. 218 p

7. Flores CR. Propiedades medicinales de la lima. Editorial Alter, México D.F.-México; 2017. 179 p.

8. Avilán LP, Rengifo CV. Los cítricos. Editorial América, Caracas-Venezuela; 1987. 248 p.

9. Arús P. The agriculture of the future: Science and technology for sustainable agricultural development. Mètode 2020;10:33-9. DOI: https://doi.org/10.7203/metode.10.12546

10.Villarroel Fuentes M. Educar para la sostenibilidad: el nuevo mantra de la educación agrícola en América Latina. IJERI. 2017;9(1):316-32.

11.Gil Pérez D, Vilches A. Ciencia de la sostenibilidad: Una revolución científica a la que la revista TED quiere contribuir. Rev Fac Cienc Tecnol 2014;36(1):7-10.

DOI: http://doi.org/10.17227/01203916.2908

12. Calvente AT. El concepto moderno de sustentabilidad. Editorial Benelux. Buenos Aires- Argentina; 2007.158 p.

13.Mazuela Aguilar PC. Agricultura sustentable. Idesia 2011;29(3):3-5. DOI: https://doi.org/10.4067/S071834292011000300001

14.Astier MA, López Ridaura S, Pérez Agis E, Masera OR. El marco de evaluación Mesmis y su aplicación en un sistema agrícola campesino en la región Purhépecha, México [Internet]. Mexico: Grupo Interdisciplinario de Tecnología Rural Apropiada A.C; 2000 [citado 26 de octubre de
2019]. Documento de Trabajo D35. Recuperado a partir de: https://www.academia.edu/30039603/Astier_and _Lopez_MESMIS

15.Scheaffer R, Mendenhall W, Ott, L. Elementos de muestreo. Editorial Iberoamericana. México D.F-México;1987. 304p.

16.Sarandón S, Marasas M, DiPietro F, Muiño A, Oscares E. Evaluación de la sustentabilidad del manejo de suelos en agroecosistemas de la provincia de La Pampa, Argentina, mediante el uso de indicadores. Rev Bras Agroecol 2006; 1(1):19-28.

17.Sarandón SJ, Zuluaga MS, Cieza R, Janjetic L, Negrete E. Evaluación de la sustentabilidad de sistemas agrícolas de fincas en Misiones, Argentina, mediante el uso de indicadores. Agroecología 2006;1:19-28.

18. Bolívar H. Metodología e indicadores de evaluación de sistemas agrícolas hacia el desarrollo sostenible CICAG 2011;8(1):1-18.

19.Pinedo Taco R, Gómez Pando L, Julca Otiniano A. Sostenibilidad de sistemas de producción de quinua (Chenopodium quinoa Willd.). Ecosistemas y Recur Agropecuarios 2018;5(15):399-409. DOI: http://doi.org/10.19136/era.a5n15.1734

20.Machado Vargas MM, Ríos Osorio LA. Sostenibilidad en agroecosistemas de café de pequeños agricultores: revisión sistemática. Idesia 2016;34(2):15-23.

DOI: https://doi.org/10.4067/S0718$\underline{34292016005000002}$

21.Silva Santamaría L, Ramírez Hernández O. Evaluación de agroecosistemas mediante indicadores de sostenibilidad en San José de las Lajas, provincia de Mayabeque, Cuba. Luna Azul 2017;(44):120-52. DOI: http://doi.org/10.17151/luaz.2017.44.8

22. Albarracín Zaidiza JA, Fonseca Carreño NE, López Vargas LH. Las prácticas agroecológicas 
como contribución a la sustentabilidad de los agroecosistemas. Caso provincia del Sumapaz. Ciencia y Agricultura 2019;16(2):39-55. DOI: http://doi.org/10.19053/01228420.v16.n2.2019.9 $\underline{139}$

23.Martínez Girón J, Marín Rivera JV, Murillo Lopera KI, Rodríguez Delgado IC. La Agroecología: alternativa de desarrollo sustentable ante la crisis ambiental en un mundo globalizado. Rev Investig Agrar Ambient 2018;9(2):63-76. DOI: https://doi.org/10.22490/21456453.2196

24.Vásquez P, Vignolles M. Establecimiento agroproductivo ecológico vs. Agricultura convencional: partido de Tandil, provincia de Buenos Aires. Soc Nat 2015;27(2):267-80. DOI: https://doi.org/10.1590/1982-451320150206

25.Casas A, Torres I, Delgado Lemus A, Rangel Landa S, Ilsley C, Torres Guevara J, et al. Ciencia para la sustentabilidad: investigación, educación y procesos participativos. Rev Mex Biodivers 2017;88(1):113-28. DOI: https://doi.org/10.1016/j.rmb.2017.10.003

26.Torres Lima PA, Cruz Castillo JG. Procesos urbanos y sistemas socioecológicos: Trayectorias sustentables de la agricultura de chinampa en Ciudad de México. Letras Verdes 2019;(25):16889.

DOI: https://doi.org/10.17141/letrasverdes.25.2019.336 $\underline{8}$

27.Álvarez AM. Retos de América Latina: Agenda para el desarrollo sostenible y negociaciones del siglo XXI. Probl Desarro 2016;47(186):9-30. Doi:

DOI: https://doi.org/10.1016/j.rpd.2016.08.002

28.Boratyńska K, Huseynov RT. An innovative approach to food security policy in developing countries. J Innovation \& Knowledge 2017;2(1): $39-44$.

DOI: https://doi.org/10.1016/j.jik.2016.01.007
29. Méndez Sastoque MJ. Ocupación rural no agrícola y desarrollo rural local: reflexiones y aportes para una articulación efectiva. Interações 2015;16(2):315-25.

DOI: https://doi.org/10.1590/151870122015207

30.Mora Rivera JJ, Cerón Monroy H. Diversificación de ingresos en el sector rural y su impacto en la eficiencia: evidencia para México. Cuad Desarro Rural 2015;12(76):57-81. DOI: https://doi.org/10,11144/Javeriana.cdr12-76.paac

31.Rodrigues Lopes G. Desmontando el desarrollo territorial rural (DTR) en América Latina. Tabula Rasa 2015;(23):181-202. DOI: https://doi.org/10.25058/20112742.46

32.Velásquez Hernández JR. Factores que inciden en el desarrollo rural en la comunidad Las Perlas, Ticuantepe, 2014. Rev Hum Cambio Soc 2015;3(6):48-60. DOI: https://doi.org/10.5377/hcs.v0i6.3521

33.Rasgado Cabrera VE, Castañeda Hidalgo E, Lozano Trejo S, Pérez León MI, Santiago Martínez GM. Sustentabilidad de agroecosistemas de maíz de la planicie costera del Istmo, Oaxaca, México. Rev Fac Agron 2019;118(2):1-12. DOI: https://doi.org/10.24215/16699513e028

34.Blanco Valdez Y. El rol de las arvenses como componente en la biodiversidad de los agroecosistemas. Cultrop 2016;37(4):34-56. DOI: https://doi.org/10.13140/RG.2.2.10964.19844

35.Santistevan Mendez MS. Sustentabilidad del cultivo del limón (Citrus aurantifolia (Christm) S.) en la provincia Santa Elena, Ecuador [tesis doctoral]. [Lima]: Universidad Nacional Agraria La Molina; 2016 [citado 26 de octubre de 2019]. Recuperado a partir de: https://alicia.concytec.gob.pe/vufind/Record/UN AL d68eb5cbfa5a5baa9568329494cce160

36.Cleves Leguízamo JA, Toro Calderón J, Martínez Bernal LF, León Sicard T. La Estructura 
agroecológica principal (EAP): novedosa herramienta para planeación del uso de la tierra en agroecosistemas. Rev Colomb Cien Hortic 2017;11(2):441-9.

DOI: https://doi.org/10.17584/rcch.2017v11i2.7350

37. Moguel P, Toledo VM. Biodiversity conservation in traditional coffee systems in Mexico. Conserv Biol 1999;13(1):11-21. DOI: https://doi.org/10.1046/j.15231739.1999.97153.x

38.Nicholls CI, Parrella M, Altieri MA. The effects of a vegetational corridor on the abundance and dispersal of insect biodiversity within a northern California organic vineyard. Landsc Ecol 2001;16(2):133-46. DOI: https://doi.org/10.1023/A:1011128222867

39.Velásquez Castro JA. Agricultura multifuncional: relevancia para el turismo en México. Revista Mexicana de Ciencias Agrícolas 2018;9(7):1494507. DOI: https://doi.org/10.29312/remexca.v9i7.1178

40.Perea Restrepo SA. Lecciones sobre permacultura para hábitat simbiótico en el paisaje cultural cafetero. Dearquitectura 2016;19(1):44-51. DOI: http://doi.org/10.18389/dearq19.2016.04

41.García S, Guerrero M. Indicadores de sustentabilidad ambiental en la gestión de espacios verdes: Parque urbano Monte Calvario, Tandil, Argentina. Rev Geogr Norte Gd 2006;(35):45-57. DOI: https://org/10.4067/S0718-34022006000100004

42. Vásquez Valencia RA, García Almada RM. Indicadores PER y FPEIR para el análisis de la sustentabilidad en el municipio de Cihuatlán, Jalisco, México. Nóesis 2018;27(54):21-31. DOI: http://doi.org/10.20983/noesis.2018.3.1

43.Eizenberg E, Jabareen Y. Social sustainability: A new conceptual framework. Sustainability 2017; 9(1):1-16.

DOI: https://doi.org/10.3390/su9010068
44.Fraser ED, Dougill AJ, Mabee WE, Reed M, Mcalpine P. Bottom up and top down: Analysis of participatory processes for sustainability indicator identification as a pathway to community empowerment and sustainable environmental management. J Environ Manage 2006;78(2): 114-27.

DOI: https://doi.org/10.1016/j.jenvman.2005.04.009

45.Gallopín GC. Environmental and sustainability indicators and the concept of situational indicators. A systems approach. Environ Model Assess 1996;1(3):101-17.

DOI: https://doi.org/10.1007/BF01874899

46. Glavic P, Lukman R. Review of sustainability terms and their definitions. J Clean Prod 2007;15(18):1875-85. DOI: https://doi.org/10.1016/j.jclepro.2006.12.006

47. Orenstein D, Shach Pinsley D. A comparative framework for assessing sustainability initiatives at the regional scale. World Dev 2017;98:245-56. DOI:

https://doi.org/10.1016/j.worlddev.2017.04.030

48.Pannell DJ, Schilizzi S. Sustainable agriculture: A matter of ecology, equity, economic efficiency or expedience?. J Sustain Agr 1999;13(4):57-66. DOI: https://doi.org/10.1300/J064v13n04 06

49.Pires A, Morato J, Peixoto H, Botero V, Zuluaga L, Figueroa A. Sustainability assessment of indicators for integrated water resources management. Sci Total Environ 2017;578:139-47. DOI: https://doi.org/10.1016/j.scitotenv.2016.10.217

50.Márquez Romero F, Julca Otiniano A, Canto Saenz M, Soplín Villacorta H, Vargas Winstanley S, Huerta Fernández P. Sustentabilidad ambiental en fincas cafetaleras después de un proceso de certificación orgánica en la convención (Cusco, Perú). Ecol Apl 2016; 15(2):125-32. DOI: http://doi.org/10.21704/rea.v15i2.752 
51.Foladori G. Sustentabilidad ambiental y contradicciones sociales. Ambiente Soc 1999; (5):1934.

DOI:

Nota del Editor:

Journal of the Selva Andina Biophere (JSAB) se mantiene neutral con respecto a los reclamos jurisdiccionales publicados en mapas y afiliaciones institucionales.

https://doi.org/10.1590/S1414753X19990002000

$\underline{03}$

52.Giraldo Díaz R, Nieto Gómez LE, Quiceno Martínez A. Evaluación de atributos de sustentabilidad de sistemas de producción campesinos en la vereda El Mesón, municipio de Palmira, Valle del Cauca (Colombia). Libre Empresa 2015; 12(1):111-35.

DOI:

https://doi.org/10.18041/libemp.v23n1.23106 